\section{TOPOLOGICAL INSULATORS}

\section{Lindblad's tenfold way}

Phys. Rev. Lett. (in the press); preprint at https:// arxiv.org/abs/1908.08834

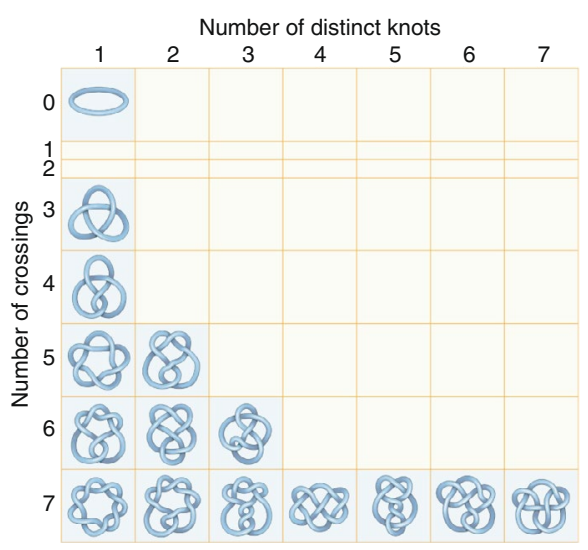

Credit: Universal Images Group North America LLC / Alamy Stock Photo

In an isolated system, symmetries are fully characterized by the system's Hamiltonian, and play an important role in determining the topology of its quantum states.

Specifically, three non-spatial symmetries of the Hamiltonian - time reversal, particlehole and chiral symmetry - sort topological insulators into ten discrete symmetry classes with distinct spectral properties. Now, Simon Lieu and co-workers have asked if a similar classification can be applied to open quantum systems.

Unlike isolated systems, the quantum state of an open system is described by a density matrix whose evolution is governed by the Lindblad equation. Therefore, the topological signatures of these states are given by the Lindblad spectrum. Lieu and co-workers found a unique way to extend the symmetries related to the spectral features of the Hamiltonian to those of the superoperator in a Lindblad equation. Topological states can then be classified into ten non-Hermitian symmetry classes labelled by these symmetries - in analogy with the isolated system.

https://doi.org/10.1038/s41567-020-0805-0

\section{ORGANIC POLARITONS}

\section{Step into the blue}

Commun. Phys. 3, 18 (2020)

High conductivity, mechanical flexibility and bandgaps in the visible frequency range make organic semiconductors choice materials for optoelectronic devices. But their charge carrier behaviour is surprisingly different from that of their inorganic counterparts; many observations remain unexplained. For example, when exciton polaritons in organic microcavities form a Bose-Einstein condensate - a state related to polariton lasing - a step-like blueshift occurs at the threshold. Through a series of experiments Timur Yagafarov and colleagues have now pin pointed the origin of this blueshift.

Having established a set of possible causes, the team systematically ruled out contributions from the Kerr effect and frequency pulling and ultimately identified an interplay of saturation effects and intermolecular energy migration as the origin of the blueshift. They further derived an analytical expression to evaluate the relative contributions of all the effects that play into the blueshift, which could help us understand non-linear light emission in organic cavities.

https://doi.org/10.1038/s41567-020-0803-2

\section{TOPOLOGICAL SUPERCONDUCTIVITY}

\section{Majorana transparency \\ Science 367, 64-67 (2020)}

In 2017, experimental evidence for onedimensional chiral Majorana fermions was shown in a topological insulator device, but has been difficult to replicate. Now, Morteza Kayyalha and co-workers have completed a comprehensive study to ascertain why.

It turns out that the interface between the topological insulator and the superconducting layer is crucial. The authors measured devices where the transport signatures and interface characteristics could be extracted at the same time. The transport signatures previously associated with the chiral Majorana states existed whenever there was a transparent interface between the topological insulator and the superconductor. This strong coupling at the interface allows the superconductor to act as a short, modifying the transport properties from the theoretical predictions.

This doesn't rule out the possibility that the old experiments observed chiral Majorana modes, but it certainly shows that more evidence is needed.

https://doi.org/10.1038/s41567-020-0801-4

\section{ECONOMIC COMPLEXITY}

\section{Concentrated cities}

Nat. Hum. Behav. https://doi.org/djtz (2020)

A graduate with a specific expertise, such as flow cytometry, could move to a city, where they will likely find more job opportunities in, say, biotechnology labs. While this choice might appear obvious, the reasons for the disproportionate concentration of economic activity in urban centres are less clear. PierreAlexandre Balland and colleagues have now found that such spatial concentration increases with the complexity of activities.

An activity is considered complex when it requires a large team of highly-skilled experts with complementary expertise. The authors analysed data on patents, occupations and industries in the United States; they used proxies for knowledge complexity - such as the size of a team responsible for a patent - and found that spatial concentration increased with the number of inventors. The connection between economic activity complexity and urban concentration could explain the spatial inequality between small and large cities.

https://doi.org/10.1038/s41567-020-0802-3

David Abergel, Elizaveta Dubrovina, Yun Li, Nina Meinzer and Stefanie Reichert 